NP17 (continued)

lated), PA (accelerometry), and diet (Perceptions of Diet at School screener). Implementation of teacher/student leader curricula is tracked through attendance/activity logs, fidelity checklists, and participant interviews. Local, state, and national dissemination planning is led by a partnership of extension agents, state department of education representatives, eXtension COP members, and WCC wellness specialists/investigators.

Evaluation: Students were $43 \%$ male, 56.5\% in elementary school, $45.8 \%$ overweight/obese, and averaged 15 minutes/day of moderate-vigorous PA at school (30 minutes/day overall). Most lunches were from the school cafeteria (50\%) or home (34\%). During lunch, 60\% reported consuming a fruit and $37 \%$ a vegetable. Six schools completed the intervention year and are in a sustainability-planning year. Of these, all WCC-teachers schools $(n=4)$ attended training, three built active wellness teams, two focused on PA opportunities and two on promoting school meals/smart snacks. Student leaders ( $n=2$ schools) focused on included advocating for PA time/promoting healthy eating through social marketing. Fifteen schools are in the intervention year with implementation tracking ongoing.

Conclusion and Implications: At the mid-point of a five-year project, schools and students within three districts have been recruited/evaluated, with intervention implementation ongoing. Two additional districts will participate in future years. Dissemination plans include train-the-trainer modules, an online professional credit course for teachers, and distributing the manualized student leader curricula to schools with active teacher wellness teams.

Funding: 2016-68001-24927.

\section{NP18 The Impact of a Comprehensive Home Food Environment Intervention for College Freshmen Living in a Residence Hall}

Abeer Almudaihim, PhD, University of Florida; Anne Mathews, PhD, RDN, University of Florida; Sarah Colby, $P h D, R D, M H S$, The University of Tennessee; Karla Shelnutt, PhD, RD, kpagan@ufl.edu, University of Florida, 572

Newell Dr, Gainesville, FL 32611

Objective: While many interventions designed to increase fruit and vegetable (FV) intake focus on increasing knowledge and changing behaviors, improving the domains of the comprehensive home food environment (HFE) (Home Food Availability and Cues; College Students' Food Practices; Social Support and Modeling) also appears to be an important target.

Determine the impact of a comprehensive HFE intervention on the HFE and FV intake of college freshmen living in a residence hall participating in the Get FRUVED study. Description: Baseline data were collected online during Fall 2016 from new freshmen $(n=149 ; 18$ to 25 years) at the University of Florida. Participants were recruited through the Get FRUVED study and listservs, lived on campus, and stored food at their dorm. Eligible participants were randomized to one of the following groups: standard Get FRUVED group (GF) or Get FRUVED plus intense comprehensive HFE intervention group (GF $+\mathrm{H})$. Surveys were completed again at post-intervention. Participants received $\$ 40$ for completing both surveys. The intervention was based on Social Cognitive Theory (SCT). The comprehensive HFE intervention included a grocery shopping tour and ten nutrition education classes that included a brief nutrition lesson, a hands-on cooking class, and a dinner discussion.

Participants completed the Comprehensive HFE Survey, Dietary Screener Questionnaire, and demographic questionnaire. Differences in HFE characteristics and FV intake between groups post-intervention were assessed using ANCOVA.

Evaluation: Seventy-five students completed pre- and post-surveys. While the HHS did not change FV intake, the intervention did result in more improved food preparation attitudes (under the HFE College Students' Food Practices Domain) in the $\mathrm{GF}+\mathrm{H}$ participants compared to GF participants at post. The lack of effect of the intervention was likely due to the poor attendance rate of the nutrition education classes.

Conclusions and Implications: Providing education on improving the comprehensive HFE may help improve certain HFE characteristics of freshmen residence halls. Research is needed to understand what motivates a student to choose to participate in campus-based nutrition education programs.

Funding: 2014-67001-21851.

\section{NP19 A Supermarket Intervention to Promote Fruit and Vegetable Consumption Among Low-Income Families}

Michele Polacsek,PhD, MHS, mpolacsek@une.edu, University of New England, 716 Stevens Ave, Portland, ME 04103; Alyssa Moran, ScD, RD, Johns Hopkins Bloomberg School of Public Health; Anne Thorndike, MPH, MD, Harvard Medical School; Rebecca Franckle, MPH, ScD, Merrimack University; Rebecca Boulos, PhD, MPH, University of Southern Maine; Aarohee Fulay, MPH, Harvard T.H. Chan School of Public Health; Jason Block, $M P H, M D$, Harvard Medical School; Eric Rimm, ScD, Harvard T.H. Chan School of Public Health

Objective: Few Americans meet national recommendations for fruit and vegetable (FV) consumption, and the high price of fresh produce is a commonly cited barrier.

We conducted a randomized controlled trial to determine if a same-day, two-for-one FV coupon (Double Bucks) combined with a point-of-purchase nutrition education program (Cooking Matters) would increase FV purchases and consumption among low-income families who were regular customers of a rural Maine supermarket.

Description: We randomized 605 parents who regularly shopped at a Maine supermarket to a six-month intervention (Double Bucks on FV up to $\$ 10 /$ day plus Cooking Matters) or a control arm. All participants were given a study loyalty card, which provided a 5\%

Continued on page $S 19$ 
NP19 (continued)

discount on groceries and tracked purchases during the three-month baseline and six-month intervention periods. The primary outcome was change in weekly spending on FV from baseline to follow-up in intervention and control groups. We also compared changes from baseline to follow-up in daily servings of FV among parents and children using a 135-item food frequency questionnaire. A baseline survey asked about the household's participation in the Supplemental Nutrition Assistance Program (SNAP), income, and food insecurity. Subgroup analyses compared intervention effects by SNAP participation.

Evaluation: At baseline, half of participants reported food insecurity. Overall, 52\% were income-eligible for SNAP, and 32\% participated in SNAP. Cooking Matters participation was low (11\%). Compared to control, the intervention group increased weekly spending on FV by $23 \%(\$ 2.23,95 \% \mathrm{CI}=\$ 1.00, \$ 3.46)$; among SNAP participants, the intervention group increased weekly spending by $43 \%(\$ 2.17,95 \% \mathrm{CI}=\$ 0.84, \$ 3.50)$. Preliminary analyses revealed no overall change in FV consumption among parents or children.

Conclusions and Implications: Supermarket financial incentives increase household purchases of FV among SNAP and non-SNAP participants, with no evidence of increased spending on other, unhealthful foods and beverages.

Funding: 2016-68001-24961.

\section{NP20 Starting Early: Expansion of a Primary Care-Based Early Child Obesity Prevention Program}

Mary Jo Messito, MD, mary.messito@nyumc.org, NYU School of Medicine, 462 First Ave, New York, NY 10016; Michelle Katzow, MS, MD, NYU School of Medicine; Alan Mendelsohn, MD, NYU School of Medicine; Rachel Gross, MS, MD, NYU School of Medicine

Objective: Determine "Starting Early Program" (StEP) impacts on infant feeding practices and weight and develop expanded prenatal and pre-school StEP interventions.

Description: We conducted a randomized controlled trial testing the efficacy of StEP, a comprehensive early child obesity prevention program using prenatal and pediatric primary care to target low-income, Hispanic families. English/Spanish speaking pregnant Hispanic women were enrolled in the third trimester to standard primary care control group vs. StEP intervention group (prenatal/postpartum nutrition counseling, and nutrition and parenting support groups coordinated with well-child visits until child age three years). Expanded prenatal and preschool StEP curricula for women beginning in the first trimester and children aged 3-5 years were developed.

Evaluation: Intervention outcomes assessed at child age 3, 10, 19 months and 2, 3, 4 and 5 years. Maternal infant feeding and activity practices assessed using 24-hour diet recalls and validated surveys. Measured child weight for age z-scores (WFAz) determined from CDC growth charts. Intent to treat analyses and within group analyses for intervention dose impacts were conducted. We randomized 533 low-income women into the original trial. 88\%, $78 \%, 74 \%, 76 \%$ and $75 \%$ of mother-infant pairs completed assessments at 3,10, and 19 months, and 2 and 3 years. Intervention pairs had healthier feeding and activity practices (more exclusive breastfeeding, tummy time; less cereal in the bottle, excess milk intake and non-responsive feeding styles). By age two, intervention group infants had lower WFAz than controls $(0.62(1.11)$ vs. $0.85(1.17) ; P=.046)$. Median number of sessions attended was $7.0(\mathrm{SD} 3.5) / 12$. High attendance reduced the odds of being overweight (AOR 0.42, $P=.02$ ). To date, a new expanded prenatal cohort is being enrolled in the 1st trimester (goal $n=200$ ); 309 pairs from the original cohort enrolled for continuation, 4- and 5-year assessments on-going.

Conclusion and Implications: StEP intervention infants had healthier feeding practices and lower weight at two years, with dose dependent reduction in overweight. Findings demonstrate a scalable system to potentially augment obesity prevention in primary care for atrisk families.

Funding: 2017-68001-26350.

\section{NP21 Advancing and Expanding HomeStyles: Shaping HOME Environments and LifeSTYLES to Prevent Childhood Obesity}

Carol Byrd-Bredbenner, PhD, RD, FAND, bredbenner@sebs. rutgers.edu, Rutgers University, 26 Nichol Ave, New Brunswick, NJ 08901; Karla Shelnutt, PhD, RDN, University of Florida; Melissa D. Olfert, DPH, RDN, West Virginia University

Objective: This multi-disciplinary, multi-institutional, multi-state, integrated research, education, and extension project will advance and expand the progress of HomeStyles toward reducing risk of childhood overweight and obesity.

Description: HomeStyles, an in-home family intervention, enables and motivates parents to shape home environments and weight-related lifestyle practices to prevent childhood obesity. Segment one of HomeStyles focused families with preschoolers (ages 2-5). A dissemination feasibility study was launched to determine how to effectively diffuse the innovations of the evidence-based HomeStyles-Segment one intervention to parents of children in preschools/daycares. The creation of a novel, culturally-competent HomeStyles-Segment two responsive to the developmentally unique needs of middle childhood (6-11 years) is in development for delivery online and in face-to-face SNAP-Ed sessions. Further, this project continues to build the expertise of the next generation of nutrition and health education professionals in creating effective childhood obesity prevention programs through formal coursework and hands-on practicums.

Evaluation: The dissemination feasibility study for Segment one includes pre/post surveys completed by a key

Continued on page $\mathrm{S} 20$ 